\title{
On the Origins of Eccentric Close-in Planets
}

\author{
Soko Matsumura and Genya Takeda and Fred A. Rasio \\ Department of Physics \& Astronomy, Northwestern University, Evanston, IL, 60208 \\ email: soko@northwestern.edu
}

\begin{abstract}
Strong tidal interaction with the central star can circularize the orbits of close-in planets. With the standard tidal quality factor $Q$ of our solar system, estimated circularization timescales for close-in extrasolar planets are typically shorter than the age of the host stars. While most extrasolar planets with orbital radii $a \lesssim 0.1 \mathrm{AU}$ indeed have circular orbits, some close-in planets with substantial orbital eccentricities have recently been discovered. This new class of eccentric close-in planets implies that either their tidal $Q$ factor is considerably higher, or circularization is prevented by an external perturbation. Here we constrain the tidal $Q$ factor for transiting extrasolar planets by comparing their circularization times with accurately determined stellar ages. Using estimated secular perturbation timescales, we also provide constraints on the properties of hypothetical second planets exterior to the known eccentric close-in planets.
\end{abstract}

\section{Introduction}

The median eccentricity of the current sample of almost 300 extrasolar planets is $\sim 0.19$, while it is 0.013 for the close-in planets with semi-major axis $a<0.1 \mathrm{AU}$. The circular orbits of close-in planets most likely result from orbital circularization due to the tidal interaction between, and energy dissipation inside such planets and the central stars (e.g., Rasio et al. 1996; Marcy et al. 1997). This requires the tidal circularization time $\tau_{\text {circ }}$ to be short compared to the age of the system $\tau_{\text {age }}$. Since $\tau_{\text {circ }}$ is a very steep function of $a$ (see Eq. 2.1 or 2.6), while $\tau_{\text {age }} \sim 1-10$ Gyr for most systems, a sharp decline in eccentricity is expected below some critical value of $a$. However, the observed transition seems to occur around $0.03-0.04 \mathrm{AU}$, whereas the calculated $\tau_{\text {circ }}$ becomes comparable to $\tau_{\text {age }}$ at $\sim 0.1 \mathrm{AU}$ (Matsumura et al. 2008). Since more than half the planets (presently $37 / 68$ ) within 0.1 AU have non-zero eccentricities, and one quarter (presently 16/68) of them have $e \geqslant 0.1$, the high eccentricities of these close-in planets demand explanation.

\section{Constraints on the tidal $Q$ value of close-in planets}

The circularization time is estimated from the rate change of eccentricity: $\tau_{\text {circ }}=-e / \dot{e}$. Here, $\dot{e}$ is the sum of the eccentricity change due to the tides raised on the star by the planet and those raised on the planet by the star. The general expression is (Goldreich \& Soter 1966; Hut 1981; Eggleton et al. 1998; Mardling \& Lin 2002):

$$
\begin{aligned}
\tau_{\text {circ }} & =\frac{2}{81} \frac{Q_{\mathrm{p}}^{\prime}}{n} \frac{M_{\mathrm{p}}}{M_{*}}\left(\frac{R_{\mathrm{p}}}{a}\right)^{-5} \\
& \times\left[\frac{Q_{\mathrm{p}}^{\prime}}{Q_{*}^{\prime}}\left(\frac{M_{\mathrm{p}}}{M_{*}}\right)^{2}\left(\frac{R_{\mathrm{p}}}{R_{*}}\right)^{-5} F_{*}+F_{\mathrm{p}}\right]^{-1}
\end{aligned}
$$

where the subscripts $\mathrm{p}$ and $*$ represent the planet and star, respectively. The modified tidal quality factor for a planet is defined as $Q_{\mathrm{p}}^{\prime} \equiv 3 Q_{\mathrm{p}} / 2 k_{\mathrm{p}}$, where $k_{\mathrm{p}}$ is the Love number, 
Table 1. Planetary and Stellar Parameters of Systems with Transiting Planets

\begin{tabular}{lccccccc}
\hline Planet ID & $M_{\mathrm{p}}\left[M_{J}\right]$ & $R_{\mathrm{p}}\left[R_{J}\right]$ & $a[\mathrm{AU}]$ & $\mathrm{e}$ & $M_{*}\left[M_{\odot}\right]$ & $R_{*}\left[R_{\odot}\right]$ & Age $[\mathrm{Gyr}]$ \\
\hline OGLE-TR-56 b & 1.29 & 1.3 & 0.0225 & 0 & 1.17 & 1.32 & $0.92(0.20-3.00)$ \\
OGLE-TR-113 b & 1.32 & 1.09 & 0.0229 & 0 & 0.78 & 0.77 & $13.28(11.00-13.92)$ \\
GJ 436 b & 0.072 & 0.38 & 0.02872 & 0.15 & 0.452 & 0.464 & $6.00(1.00-10.00)$ \\
OGLE-TR-132 b & 1.14 & 1.18 & 0.0306 & 0 & 1.26 & 1.34 & $0.96(0.12-3.84)$ \\
HD 189733 b & 1.15 & 1.156 & 0.0312 & 0 & 0.8 & 0.753 & $8.96(1.04-13.72)$ \\
TrES-2 & 1.98 & 1.22 & 0.0367 & 0 & 0.98 & 1 & $5.10(2.40-7.80)$ \\
WASP-14 b & 7.725 & 1.259 & 0.037 & 0.095 & 1.319 & 1.297 & $0.75(0.5-1)$ \\
WASP-10 b & 3.06 & 1.29 & 0.0371 & 0.057 & 0.71 & 0.783 & $0.8(0.6-1)$ \\
HAT-P-3 b & 0.599 & 0.89 & 0.03894 & 0 & 0.936 & 0.824 & $0.40(0.10-6.90)$ \\
TrES-1 & 0.61 & 1.081 & 0.0393 & 0 & 0.87 & 0.82 & $11.40(3.20-13.84)$ \\
HAT-P-5 b & 1.06 & 1.26 & 0.04075 & 0 & 1.16 & 1.167 & $2.60(0.80-4.40)$ \\
OGLE-TR-10 b & 0.63 & 1.26 & 0.04162 & 0 & 1.18 & 1.16 & $1.20(0.16-4.64)$ \\
HD 149026 b & 0.36 & 0.71 & 0.0432 & 0 & 1.3 & 1.45 & $2.00(1.20-2.80)$ \\
HAT-P-4 b & 0.68 & 1.27 & 0.0446 & 0 & 1.26 & 1.59 & $4.20(3.60-6.80)$ \\
HD 209458 b & 0.69 & 1.32 & 0.045 & 0 & 1.01 & 1.12 & $4.00(2.00-6.00)$ \\
OGLE-TR-111 b & 0.53 & 1.067 & 0.047 & 0 & 0.82 & 0.831 & $5.17(0.17-13.41)$ \\
HAT-P-6 b & 1.057 & 1.33 & 0.05235 & 0 & 1.29 & 1.46 & $2.30(1.60-2.80)$ \\
HAT-P-1 b & 0.524 & 1.36 & 0.0553 & 0.067 & 1.133 & 1.115 & 3.60 \\
HAT-P-2 b & 8.64 & 0.952 & 0.0677 & 0.517 & 1.298 & 1.412 & $2.70(1.30-4.10)$ \\
\hline
\end{tabular}

Data are from http://exoplanet.eu/. For systems with enough data available, ages are computed using the stellar evolution database in Takeda et al. (2007). Median values of the derived posterior age probability distribution functions are presented here, together with the $95 \%$ credible intervals in parenthesis.

and $Q_{\mathrm{p}}$ is the specific dissipation function that depends on the planetary structure as well as the frequency and amplitude of tides. Also, $F_{*}$ and $F_{p}$ are

$$
\begin{aligned}
& F_{*}=\left[f_{1}\left(e^{2}\right)-\frac{11}{18} f_{2}\left(e^{2}\right) \frac{\Omega_{*, \text { rot }}}{n}\right] \\
& F_{\mathrm{p}}=\left[f_{1}\left(e^{2}\right)-\frac{11}{18} f_{2}\left(e^{2}\right) \frac{\Omega_{\mathrm{p}, \text { rot }}}{n}\right],
\end{aligned}
$$

where $n=\sqrt{G\left(M_{*}+M_{\mathrm{p}}\right) / a^{3}}$ is the mean motion, $\Omega_{\text {rot }}$ is the rotational frequency, and

$$
\begin{aligned}
& f_{1}\left(e^{2}\right)=\left(1+\frac{15}{4} e^{2}+\frac{15}{8} e^{4}+\frac{5}{64} e^{6}\right) /\left(1-e^{2}\right)^{13 / 2}, \\
& f_{2}\left(e^{2}\right)=\left(1+\frac{3}{2} e^{2}+\frac{1}{8} e^{4}\right) /\left(1-e^{2}\right)^{5} .
\end{aligned}
$$

Generally $F_{*}$ and $F_{p}$ are comparable, and thus the effect of the stellar damping on the planetary orbital eccentricity is negligible unless the planet-to-star mass (radius) ratio is large (small) or $Q_{*}^{\prime} \ll Q_{p}^{\prime}$. We define the circularization time due to damping in the planet alone as

$$
\tau_{\text {circ }, 0}=\frac{2}{81} \frac{Q_{\mathrm{p}}^{\prime}}{n} \frac{M_{\mathrm{p}}}{M_{*}}\left(\frac{R_{\mathrm{p}}}{a}\right)^{-5} F_{\mathrm{p}}^{-1} .
$$

Note that $\tau_{\text {circ }, 0}$ can be shorter or longer than $\tau_{\text {circ }}$, depending on the sign of $F_{*}$, which changes at $\left(\Omega_{* \text {,rot }} / n\right)_{\text {crit }}=18 / 11\left(f_{1} / f_{2}\right)$. In the limit $e \rightarrow 0$, this equation leads to the standard expression for the circularization time (Eq. 4.198 of Murray \& Dermott 1999).

Now we use Equation 2.1 and 2.6 to place constraints on the tidal $Q$ values of the transiting planets, whose properties are summarized in Table $1 \dagger$. An upper limit on $Q$

$\dagger$ Note that, in Eq. 2.1, it is implicitly assumed that the star and the planet both have zero obliquity. Currently available measurements of the Rossiter-MacLaughlin effect shows that the planetary orbits in general are closely aligned with the stellar equator (Queloz et al. 2000; Winn et al. 2005). Current exceptions may be the HD 17156 and XO-3 systems (Narita et al. 2007; Hebrard et al. 2008), which we exclude from our analysis. 
is provided for planets with circular orbits since the circularization must have occurred within the lifetime of the systems $\left(\tau_{\text {circ }}<\tau_{\text {age }}\right)$. Our assumption here is that these close-in planets formed through tidal circularization of initially eccentric orbits. Nagasawa et al. (2008) showed that about one third of multiple planetary systems could form close-in planets through tidal circularization following a large eccentricity gain through planetplanet scattering or Kozai-type perturbations. Direct observational evidence suggests that a hypothesis of initially large orbital eccentricities for close-in planets is supported by the absence of planetary orbits within twice the Roche limit around the star (Faber et al. 2005; Ford \& Rasio 2006). On the other hand, close-in, eccentric planets impose a lower limit on $Q$ values, since $\tau_{\text {circ }} \geqslant \tau_{\text {age }}$ is expected for these systems. The underlying assumption is that they are not currently subject to any eccentricity excitation mechanism. We will discuss the possibilities of such mechanisms in the next section.

In calculating these limits, we have to specify three variables, namely $\Omega_{\mathrm{p}, \mathrm{rot}}, \Omega_{*, \mathrm{rot}}$, and $Q_{*}^{\prime}$. For $\Omega_{\mathrm{p}, \text { rot }}$, when planets are on circular orbits, we assume that they are perfectly synchronized, i.e., $\Omega_{\mathrm{p} \text {,rot }} / n=1$, since the spin-orbit synchronization times are $\sim 10^{-3} \tau_{\text {circ }}$ (Rasio et al. 1996). On the other hand, when planets are on eccentric orbits, they should spin down until they reach quasi-synchronization (Dobbs-Dixon et al. 2004). For these eccentric planets, we adopt a planetary spin frequency such that the rate of change of spin frequency is zero (Eq. 54 of Mardling \& Lin 2002). As for the stellar spin, the periods derived from the observed $v_{\text {rot }} \sin i$ range over $P_{* \text {,rot }} \sim 3-70$ days (Barnes 2001). Therefore, we assume that the stellar rotation of the transiting planets fall in this range.

For $P_{*, \text { rot }} \sim 3-70$ days, all planets in Table 1 take $\Omega_{*, \text { rot }} / n<\left(\Omega_{*, \text { rot }} / n\right)_{\text {crit }}$, and hence $F_{*}>0$ and $\tau_{\text {circ }, 0}>\tau_{\text {circ }}$. For planets with equivalent to zero (non-zero) eccentricity, we require $\tau_{\text {circ }}<\tau_{\text {circ }, 0}<\tau_{\text {age }}\left(\tau_{\text {age }}<\tau_{\text {circ }}<\tau_{\text {circ }, 0}\right)$. In other words, we assume that zero (non-zero) eccentricity planets have been (have not been) circularized within the lifetime of the system, independent of the rotation period of the star. This gives the upper and lower limit for circular and eccentric planets, respectively, as follows:

$$
\begin{gathered}
Q_{\mathrm{p}}^{\prime}<\frac{81}{2} n\left(\frac{M_{*}}{M_{\mathrm{p}}}\right)\left(\frac{R_{\mathrm{p}}}{a}\right)^{5} F_{\mathrm{p}} \tau_{\text {age }} \equiv Q_{\mathrm{p}, \text { crit }}^{\prime}, \\
Q_{\mathrm{p}}^{\prime}>Q_{\mathrm{p}, \text { crit }}^{\prime}\left(1-\frac{81}{2} \frac{n}{Q_{*}^{\prime}}\left(\frac{M_{\mathrm{p}}}{M_{*}}\right)\left(\frac{R_{*}}{a}\right)^{5} F_{*} \tau_{\text {age }}\right)^{-1} .
\end{gathered}
$$

The latter condition also gives the lower limit for the stellar tidal $Q$ factor, since the denominator must be positive:

$$
Q_{*}^{\prime}>\frac{81}{2} n\left(\frac{M_{\mathrm{p}}}{M_{*}}\right)\left(\frac{R_{*}}{a}\right)^{5} F_{*} \tau_{\text {age }} \equiv Q_{*, \min }^{\prime} .
$$

This corresponds to a minimum stellar $Q$ value of $Q_{*}^{\prime} \sim 2.7 \times 10^{4}-3.9 \times 10^{7}$, with a median value of $0.4-1 \times 10^{6}$ for $P_{*, \text { rot }}=3-70$ days. These values are within the range of theoretical expectations (e.g. Terquem et al. 1998; Carone \& Pätzold 2007).

Figure 1 shows the upper and lower limits for the planets' tidal $Q$ values as a function of semi-major axis for planets on circular and eccentric orbits, respectively. Since the lower limits on $Q$ values for eccentric planets depend on $Q_{*}^{\prime}$, we take three different cases of $Q_{*}^{\prime}=2,5$, and $10 Q_{* \text {,min }}^{\prime}$ as examples. Note that, with such a definition of $Q_{*}^{\prime}, Q_{\mathrm{p}}^{\prime}$ in Eq. 2.8 becomes independent of the stellar spin rate.

Since circularization times are shorter for planets with smaller orbital radii, we tend to overestimate the maximum $Q$ values at the shortest-period end. It appears that all transiting planets must have tidal $\mathrm{Q}$ values in the range $10^{5} \lesssim Q_{\mathrm{p}}^{\prime} \lesssim 10^{9}$. The figure also 


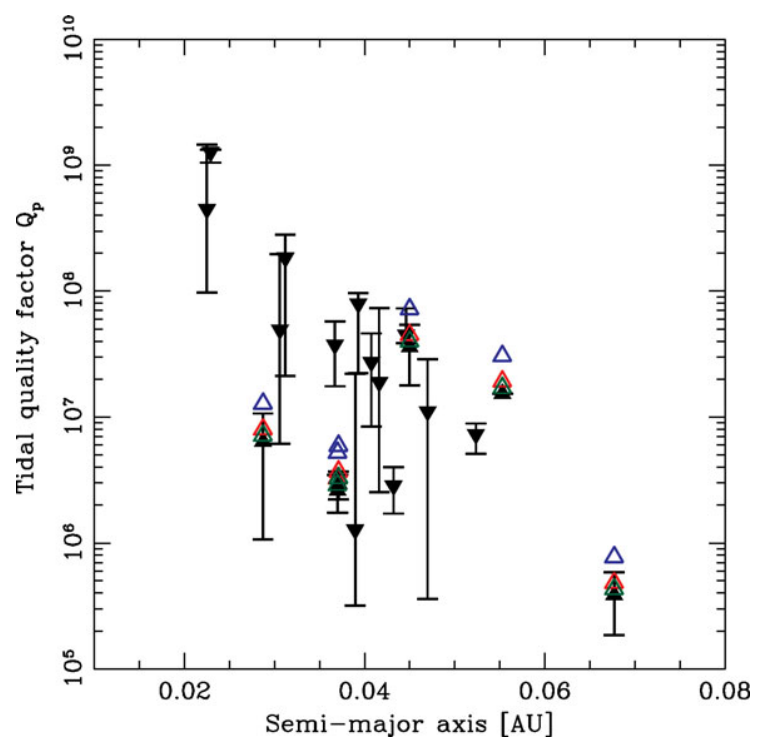

Figure 1. Estimated tidal $Q$ factors for the case of slowly rotating stars $\left(\Omega_{*} / n<\left(\Omega_{*} / n\right)_{\text {crit }}\right)$. Upper/lower limits calculated from Eq. 2.6 are shown in black down/up triangles for planets with zero/non-zero eccentricities. Open triangles are the corresponding estimates from Eq. 2.1, which approach black ones as we take 2, 5, and 10 times the minimum stellar tidal $Q$ s that are obtained from Eq. 2.9.

shows that the high eccentricities of some planets (marked with upper triangles) can be explained by assuming rather large $\left(Q_{\mathrm{p}}^{\prime} \gtrsim 10^{6}\right)$ but reasonable $\left(\lesssim 10^{9}\right)$ tidal $Q$ values.

Although these estimated $Q$ values may be larger than those of Jupiter $\left(1.82 \times 10^{5} \leqslant\right.$ $Q_{\mathrm{J}}^{\prime} \leqslant 6.07 \times 10^{5}$, Yoder \& Peale 1981) or Neptune $\left(3.3 \times 10^{4} \leqslant Q_{\mathrm{N}}^{\prime} \leqslant 1.2 \times 10^{6}\right.$, Banfield \& Murray 1992; Zhang \& Hamilton 2007), these higher values cannot be excluded. Recent studies of the excitation and dissipation of dynamical tides within rotating giant planets have shown that tidal $Q$ values fluctuate strongly depending on the tidal forcing frequency, and the effective $Q$ values could go up to $\sim 10^{9}$ depending on the spin rate as well as the internal structure of the planet (e.g., presence/absence of a core, radiative envelope, or a density jump, see Ogilvie \& Lin 2004; Wu 2005). According to these detailed models, it remains possible that some planets maintain large eccentricities simply because of their larger $Q$ values.

\section{Dynamical Perturbations}

Candidate perturbation mechanisms that could excite and maintain planetary eccentricities include (1) tidal interaction with the central star (Dobbs-Dixon et al. 2004), (2) quadrupole or higher-order secular perturbation from an additional body, or (3) resonant interaction with another planet. Here we focus on (2). For the discussion of (1), and (3), see Matsumura et al. (2008).

When there is a large mutual inclination angle $\left(i \gtrsim 40^{\circ}\right)$ between the two planets, Kozai-type perturbations can become important (Kozai 1962). Such highly non-coplanar planetary orbits could result from planet-planet scattering after dissipation of the gaseous disk. Chatterjee et al. (2007); Nagasawa et al. (2008) have performed extensive numerical scattering experiments and showed that the final inclination of planets could be as high as $70^{\circ}$, with a median of $10-20^{\circ}$. On the other hand, when a mutual inclination angle 


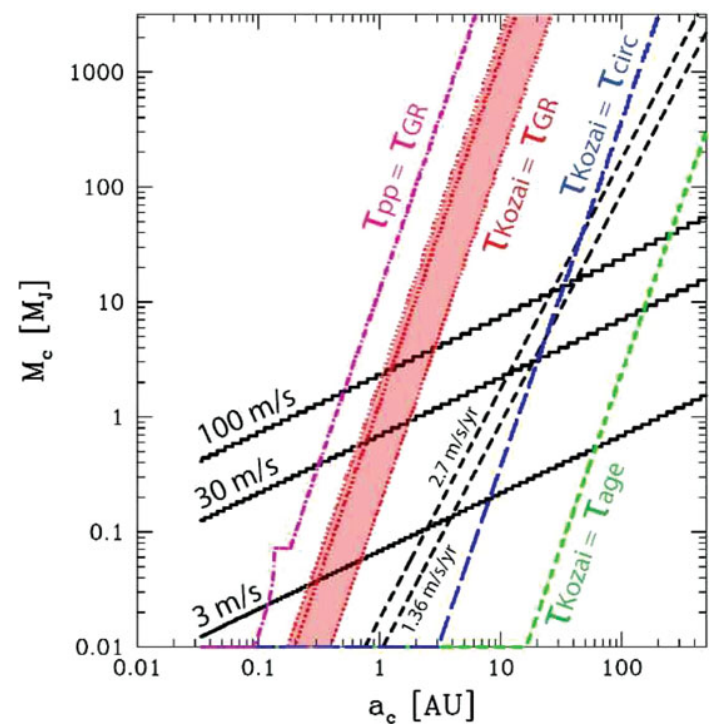

Figure 2. Various secular eccentricity excitation timescales for the planet GJ $436 \mathrm{~b}$ caused by a hypothetical planetary (or stellar) companion GJ $436 \mathrm{c}$ with mass $M_{\mathrm{c}}$ and semi-major axis $a_{\mathrm{c}}$. The solid black lines show the predicted radial velocity amplitudes caused by the undetected companion. The dot-dashed line shows the threshold right of which the secular interaction between planets with $i \lesssim 40 \mathrm{deg}$ is suppressed by GR precession, while the three red dotted lines are the similar thresholds for the Kozai mechanism with the assumed orbital eccentricity of the companion $e_{\mathrm{c}}=0.01,0.5$, and 0.9 from left to right. Thresholds for $\tau_{\text {circ }}$ and $\tau_{\text {age }}$ are shown for comparison. Also plotted are the linear trends from (Butler et al. 2004; Maness et al. 2007).

is smaller $\left(i \lesssim 40^{\circ}\right)$, octupole perturbations may still moderately excite the eccentricity of the close-in planet. The secular interaction timescale of a pair of planet with small mutual inclination can be derived from the classical Laplace-Lagrange theory (Brouwer \& Clemence 1961; Murray \& Dermott 1999).

For this secular perturbation from an additional planet to be causing the large eccentricity of the close-in planet, it must occur fast enough compared to other perturbations causing orbital precession. In particular, GR precession and tides are important effects that would compete against the perturbation from the additional body (Holman et al. 1997)†. GR precession, Kozai eccentricity oscillations, and the secular perturbation from a low-inclination perturber occur on the following timescales: (Kiseleva et al. 1998; Fabrycky \& Tremaine 2007; Zhou \& Sun 2003):

$$
\begin{aligned}
\tau_{\mathrm{GR}} & =\frac{2 \pi c^{2} a_{\mathrm{p}}}{3 G\left(M_{*}+M_{\mathrm{p}}\right) n_{\mathrm{p}}}\left(1-e_{\mathrm{p}}^{2}\right) \\
\tau_{\text {Kozai }} & =\frac{4 n_{\mathrm{p}}}{3 n_{\mathrm{c}}^{2}}\left(\frac{M_{*}+M_{\mathrm{p}}+M_{\mathrm{c}}}{M_{\mathrm{c}}}\right)\left(1-e_{\mathrm{c}}^{2}\right)^{3 / 2} \\
\tau_{\mathrm{pp}} & =\frac{4 \pi}{\left(c_{1}+c_{2}\right) \pm \sqrt{\left(c_{1}-c_{2}\right)^{2}+4 c_{0}^{2} c_{1} c_{2}}},
\end{aligned}
$$

$\dagger$ Although stellar and planetary rotational distortions cause additional pericenter precessions (Sterne 1939), GR precession dominates unless the stellar rotation is on the high end. 
where the subscript $\mathrm{c}$ indicates the companion body, $c$ is the speed of light, and in $\tau_{\mathrm{pp}}$,

$$
\begin{aligned}
c_{0} & =b_{3 / 2}^{(2)}\left(\frac{a_{\mathrm{p}}}{a_{\mathrm{c}}}\right) / b_{3 / 2}^{(1)}\left(\frac{a_{\mathrm{p}}}{a_{\mathrm{c}}}\right), \\
c_{1} & =\frac{1}{4} n_{\mathrm{p}} \frac{M_{\mathrm{c}}}{M_{*}+M_{\mathrm{p}}}\left(\frac{a_{\mathrm{p}}}{a_{\mathrm{c}}}\right)^{2} b_{3 / 2}^{(1)}\left(\frac{a_{\mathrm{p}}}{a_{\mathrm{c}}}\right), \\
c_{2} & =\frac{1}{4} n_{\mathrm{c}} \frac{M_{\mathrm{p}}}{M_{*}+M_{\mathrm{c}}}\left(\frac{a_{\mathrm{p}}}{a_{\mathrm{c}}}\right) b_{3 / 2}^{(1)}\left(\frac{a_{\mathrm{p}}}{a_{\mathrm{c}}}\right),
\end{aligned}
$$

with $b_{3 / 2}^{(i)}\left(a_{\mathrm{p}} / a_{\mathrm{c}}\right)(i=1,2)$ being the standard Laplace coefficients.

Figure 2 illustrates the constraints on the mass and orbital radius of the hypothetical secondary planet in the GJ 436 system, which are set by comparing the GR precession with secular timescales. These constraints are qualitatively similar for the other systems with eccentric close-in planets. Generally GR precession occurs faster than any other perturbation mechanism, and therefore provides the most stringent constraint on the properties of the hypothetical planet. In order to induce the Kozai cycles of the inner planet despite the GR precession (left of the dotted lines) while not causing radialvelocity amplitude above the detection limit of $\sim 5 \mathrm{~ms}^{-1}$ (below black lines), the mass upper limit of the hypothetical planet is $\sim 1 M_{\text {Neptune }}$. For near-coplanar systems even tighter constraints are placed on the properties of the secondary planet, since they have to be on the lefthand side of the dot-dashed line.

However, there are some caveats here. For one thing, this diagram only rules out the possibility of hypothetical planets on the right of the dot-dashed/dashed lines which are currently able to oscillate the eccentricity of GJ 436 b. For example, a possible second planet, which is expected from the linear trend seen in the radial velocities (Butler et al. 2004; Maness et al. 2007, plotted as dashed lines in Fig. 2), cannot oscillate the eccentricity of GJ $436 \mathrm{~b}$, but this does not discard the possibility that such a hypothetical planet was interacting with GJ $436 \mathrm{~b}$ in the past. For another, while slowly-occurring Kozai-type perturbations are almost always suppressed by GR precession, eccentricity excitation through secular octupole perturbation may be occasionally enhanced by GR effects (Ford et al. 2000; Adams \& Laughlin 2006). For the case of the GJ 436 system, we have numerically tested the effect of a hypothetical secondary planet c on the eccentricity evolution of the inner planet $\mathrm{b}$. We have found that, within the detectable radial-velocity limit $\lesssim 5 \mathrm{~m} / \mathrm{s}$, a planet $\mathrm{c}$ cannot excite the eccentricity of the planet b from 0.01 to the observed 0.15 , even if its eccentricity is as large as 0.5 . This result holds for other systems since they have an even heavier planet. Therefore, we can safely exclude the possibility that these planets obtain their current high eccentricities through secular perturbation from an undetected planet with up to Neptune's mass after their orbits are circularized.

\section{Summary}

In this proceedings, we have investigated the origins of close-in planets on an eccentric orbit. We place constraints on the tidal Q factor of transiting planets by comparing the stellar age with the tidal circularization time, and find that $10^{5} \lesssim Q_{\mathrm{p}}^{\prime} \lesssim 10^{9}$, which agrees well with current theoretical estimates, can explain these eccentric planets. We also show that it is difficult to explain the high eccentricities of these planets by invoking an interaction with an unseen second planet. Our results suggest that at least some of the close-in eccentric planets may be simply in the process of getting circularized. 


\section{Acknowledgements}

This work was supported by NSF Grant AST-0507727.

\section{References}

Adams, F. C. \& Laughlin, G. 2006, ApJ, 649, 992

Banfield, D. \& Murray, N. 1992, Icarus, 99, 390

Barnes, S. A. 2001, ApJ, 561, 1095

Brouwer, D. \& Clemence, G. M. 1961, Methods of celestial mechanics (New York: Academic Press, 1961)

Butler, R. P., Vogt, S. S., Marcy, G. W., Fischer, D. A., Wright, J. T., Henry, G. W., Laughlin, G., \& Lissauer, J. J. 2004, ApJ, 617, 580

Carone, L. \& Pätzold, M. 2007, P\&SS, 55, 643

Chatterjee, S., Ford, E. B., \& Rasio, F. A. 2007, ArXiv Astrophysics e-prints

Dobbs-Dixon, I., Lin, D. N. C., \& Mardling, R. A. 2004, ApJ, 610, 464

Eggleton, P. P., Kiseleva, L. G., \& Hut, P. 1998, ApJ, 499, 853

Faber, J. A., Rasio, F. A., \& Willems, B. 2005, Icarus, 175, 248

Fabrycky, D. \& Tremaine, S. 2007, ApJ, 669, 1298

Ford, E. B., Kozinsky, B., \& Rasio, F. A. 2000, ApJ, 535, 385

Ford, E. B. \& Rasio, F. A. 2006, ApJL, 638, L45

Goldreich, P. \& Soter, S. 1966, Icarus, 5, 375

Hebrard, G., Bouchy, F., Pont, F., Loeillet, B., Rabus, M., Bonfils, X., Moutou, C., Boisse, I., Delfosse, X., Desort, M., Eggenberger, A., Ehrenreich, D., Forveille, T., Lagrange, A. M., Lovis, C., Mayor, M., Pepe, F., Perrier, C., Queloz, D., Santos, N. C., Segransan, D., Udry, S., \& Vidal-Madjar, A. 2008, A\&A 488, 763

Holman, M., Touma, J., \& Tremaine, S. 1997, Nature, 386, 254

Hut, P. 1981, A\&A, 99, 126

Kiseleva, L. G., Eggleton, P. P., \& Mikkola, S. 1998, MNRAS, 300, 292

Kozai, Y. 1962, AJ, 67, 591

Maness, H. L., Marcy, G. W., Ford, E. B., Hauschildt, P. H., Shreve, A. T., Basri, G. B., Butler, R. P., \& Vogt, S. S. 2007, PASP, 119, 90

Marcy, G. W., Butler, R. P., Williams, E., Bildsten, L., Graham, J. R., Ghez, A. M., \& Jernigan, J. G. 1997, ApJ, 481, 926

Mardling, R. A. \& Lin, D. N. C. 2002, ApJ, 573, 829

Matsumura, S., Takeda, G., \& Rasio, F. A. 2008, submitted

Murray, C. D. \& Dermott, S. F. 1999, Solar system dynamics (Solar system dynamics by Murray, C. D., 1999)

Nagasawa, M., Ida, S., \& Bessho, T. 2008, ArXiv e-prints, 801

Narita, N., Sato, B., Ohshima, O., \& Winn, J. N. 2007, ArXiv e-prints, 712

Ogilvie, G. I. \& Lin, D. N. C. 2004, ApJ, 610, 477

Queloz, D., Eggenberger, A., Mayor, M., Perrier, C., Beuzit, J. L., Naef, D., Sivan, J. P., \& Udry, S. 2000, A\&A, 359, L13

Rasio, F. A., Tout, C. A., Lubow, S. H., \& Livio, M. 1996, ApJ, 470, 1187

Sterne, T. E. 1939, MNRAS, 99, 451

Takeda, G., Ford, E. B., Sills, A., Rasio, F. A., Fischer, D. A., \& Valenti, J. A. 2007, ApJS, 168, 297

Terquem, C., Papaloizou, J. C. B., Nelson, R. P., \& Lin, D. N. C. 1998, ApJ, 502, 788

Winn, J. N., Noyes, R. W., Holman, M. J., Charbonneau, D., Ohta, Y., Taruya, A., Suto, Y., Narita, N., Turner, E. L., Johnson, J. A., Marcy, G. W., Butler, R. P., \& Vogt, S. S. 2005, ApJ, 631, 1215

Wu, Y. 2005, ApJ, 635, 688

Yoder, C. F. \& Peale, S. J. 1981, Icarus, 47, 1

Zhang, K. \& Hamilton, D. P. 2007, submitted to Icarus

Zhou, J.-L. \& Sun, Y.-S. 2003, ApJ, 598, 1290 